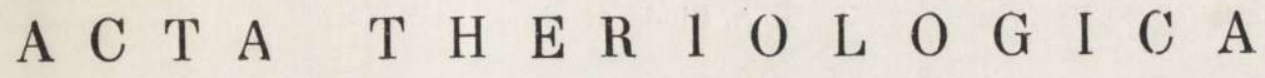 \begin{tabular}{lll} 
VOL. XIV, 18: 247-262. BIAŁOWIEŻA & 30.VIII.1969 \\
\hline
\end{tabular}
}

\author{
Andrzej S I UDA, Wirgiliusz ŻUROWSKI \& Hanna SIUDA
}

\section{The Food of the Roe Deer}

[With 3 Tables]

\begin{abstract}
A total of 46 rumens of roe-deer, Capreolus capreolus (L i n n a e us, 1758) were examined over the course of four hunting seasons from 1963-1966. The material was obtained from part of the Pisz Forest $\left(\left(53^{\circ} 43^{\prime} \mathrm{N}, 21^{\circ} 36^{\prime} \mathrm{E}\right)\right.$, where the Pineto-Vaccinietum myrtilli and PinetoQuercetum associations dominated. 178 species of plants were identified in the rumen contents. Seasonal variations were found in the food of roe-deer. Their basic food consists of the leaves and twigs of trees, shrubs and dwarf-shrubs. During spring herbs played an important part in their food. Grasses and sedges appeared in the food in small numbers during all the year. From 9 to 42 species of plants were identified in individual rumens, and of these one or at most three species dominated. The following species dominated in spring: Quercus sp., Fragaria vesca L., Melampyrum pratense L., Chamaenerion angustifolium (L.) Scop., Carpinus betulus L.; in summer: Rubus idaeus L., Vaccinium myrtillus L., Quercus sp., Salix caprea L., Betula verrucosa Ehrh.; in autumn: Calluna vulgaris (L.) Salisb., Rubus idaeus L., Vaccinium myrtillus L., Pinus silvestris L., Betula verrucosa Ehrh.; and in winter: Pinus silvestris L., Calluna vulgaris (L.) Salisb., Vaccinium myrtillus L., Picea excelsa (Lam) Lk., Vaccinium vitis-idaea L.
\end{abstract}

\section{MATERIALS AND METHODS}

Studies on the food of roe-deer were undertaken for the purpose of ascertaining what factors determine the quality of the roe-deer and its antlers, what influence the roe-deer exerts on its environment and what game management is required in order to limit to a minimum the damage done by roe-deer. Roe-deer live in very varied habitats, hence the necessity for carrying out numerous observations and for referring conclusions to definite geographical regions.

The method of botanical analysis of the rumen contents was used in these investigations. The material studied consisted of the contents of the rumen from roe-deer shot during the period 1963-1966 in the Experimental Hunting Range at Popielno in the Olsztyn province. The Range is situated in the Pisz Forest $\left(53^{\circ} 43^{\prime} \mathrm{N}, 21^{\circ} 36 \mathrm{E}\right)$ on a peninsula between lakes Beldany, Sniardwy and Warnolty. 
The total area of the Range is 2211 ha, of which $69 \%$ is covered by forest, $7 \%$ by meadows and pastures, $5 \%$ by arable land, $17 \%$ by lakes, $1 \%$ by peat bogs and $1 \%$ by buildings. $85 \%$ of the wooded area is covered by the associations Pineto-Vaccinietum myrtilli (Braun-Blanquet \& Vlieger, 1939) and Pineto-Quercetum (K o z low s k a, 1925).

A large number of animals were maintained in the Range for research purposes: about 35 red deer, about 60 roe-deer and about 35 wild boar. In addition there were about 30 wild Polish ponies. All the animals were kept supplied with supplementary food during the winter.

The shot roe-deer were in good condition. The average weight of adult bucks before eviscerating was $25.1 \mathrm{~kg}$, of does $23.8 \mathrm{~kg}$, and after gralloching respectively: $17.8 \mathrm{~kg}$ and $16.4 \mathrm{~kg}$. Body weight was similar to the data given by $\mathrm{R}$ a e sfeld (1965) for roe-deer from the Mazurian Lake District, and was greater than Guz iń s k i's data for Mazuria (1964).

Forty six rumens were used for botanical analysis (Table 1). The rumen contents were washed on a fine sieve of about $1 \mathrm{~mm}^{2}$ mesh and the residue preserved in $5 \%$ formalin solution. Samples were kept in hermetically closed jars. Before beginning the analysis the material was spread evenly over a flat dish, from which a total amount of $100 \mathrm{ml}$ per sample was taken from places at regular intervals (modifying $\mathrm{Ch}$ amrad \& Box's method, 1964). The rumen contents,

Table 1.

Particulars of animals used for investigations in different seasons.

\begin{tabular}{|c|c|c|c|c|c|c|}
\hline Season & $\sigma_{\sigma^{x} \sigma^{x}}^{\text {Number }}$ & of animals & $\begin{array}{l}\text { Average } \\
\sigma^{x} \sigma^{x}\end{array}$ & $\begin{array}{c}\text { age in years } \\
\text { o }+9\end{array}$ & $\begin{array}{l}\text { Average body } \\
0^{x} \sigma^{7}\end{array}$ & $\begin{array}{l}\text { weight.kg } \\
\text { 우 }\end{array}$ \\
\hline Spring & 9 & - & 35 & - & 24.2 & - \\
\hline Summer & 6 & 3 & 5.7 & 3.3 & 25.0 & 238 \\
\hline Autumn & 3 & 4 & 3.7 & 4.6 & 26.8 & 21.5 \\
\hline Winter & 3 & 18 & 3.0 & 3.3 & 23.0 & 20.9 \\
\hline
\end{tabular}

carefully stored as above, retained their fresh appearance, natural colour, thickness, stiffness, hairs on the leaves and similar morphological characters. In the rinsed fraction we found the terminal parts of branches, sometimes several centimeters in length, with characteristic buds, bark, leaf cicatricles, stigmata, whole small leaves and large fragments of bigger leaves, stipules, climbing tendrils, flowers, fruits, seeds, ears and blades of grasses. Atlases and keys for identifying plants, and herbarium specimens collected in the study area were used for identifying the elements of the animals' food ( $\mathrm{Sz}$ a fer et al, 1953; K u le$\mathrm{sza}, 1952$; C zarnocki, 1950). Greatly triturated and partly digested particles were identified mainly on the basis of characteristic details of the leaf epidermis and ligneous or bark elements, using microtechnical and plant anatomy methods. During analysis the larger and more characteristic fragments of plants were identified macroscopically, while the remainder were identified by means of a stereoscopic microscope. Fragments of plants of different species were segregated into separate dishes. After completing segregation excess water was drained off, and then the volume of the segregated material measured in a measuring vessel filled with water, with accuracy to $0.1 \mathrm{ml}$. Calculation was made on this basis of the percentage of different species of plants in the rumen contents. Species 
occupying less than $1 \%$ of the volume of the sample were given the symbol $"+$ «. Four periods were accepted, for which frequency and the quantitative participation of different species of plants in the rumen contents were determined: spring, summer, autumn and winter (Table 2).

Using the methods adopted by Anderson et al. (1965) and Kolev (1966) as a guide, calculation was made of the index of importance of different species of plants. The index is the quotient of frequency and mean percentage of volume of the given species in all samples for the season.

\section{RESULTS AND DISCUSSION}

The rumen contents weight from $1.2 \mathrm{~kg}$ to $3.2 \mathrm{~kg}$, with an average of $1.7 \mathrm{~kg}$. The mean weight of the rumens examined by $\mathrm{M}$ ot tl (1957) was $1.6 \mathrm{~kg}$, and he considered that this amount corresponded to the daily portion of food consumed by a roe-deer. Ne č as (1963) states that according to $\mathrm{L} \mathrm{oc} \mathrm{hman}$ the roe-deer consumes about $4.0 \mathrm{~kg}$ of green food daily. The rumen contents we examined contained from $11 \%$ to $14 \%$ of air-dry mass.

A total of 178 species of plants were identified. The species composition varied over the course of the year, such variations being connected with the season.

\section{Spring Period (March 22nd - June 21st)}

During this period the material was collected from roe-deer shot between May 31st and June 21st (Table 2). Unidentified particles formed about $10 \%$ of the sample. From 15 to 42 species of plants were identified in one rumen. A total of 102 species were identified in rumens at this period. Leaves and young twigs of trees represent on an average $38 \%$ of the rumen contents. Quercus robur L., which attained $100 \%$ frequency, dominated and in some cases formed $60 \%$ of the volume of the rumen, with an average of $27 \%$. In addition to Quercus the following plants were eaten: Carpinus betulus L., Populus tremula L., Betula verrucosa Ehrh. The considerable percentage of birch in the food was probably due to the commonness of its occurrence in the area, and not to its attractive taste. Acer platanoides L., which occurs only sporadically in the forest, was however attractive to these roe-deer. In some rumens its contents formed several per cent of the volume.

In spring herb species formed about $40 \%$ of the roe-deer's food. These were usually species easy to find and growing luxuriantly. Among the most important were: Fragaria vesca L., Melampyrum pratense L., Chamaenerion angustifolium (L.) Scop. and various species of the genera: Potentilla L., Ranunculus L., Galium L., Plantago L., Veronica L., Rumex L. 
Of the group of shrubs and dwarf-shrubs Rubus idaeus L. formed the largest percentage in the roe-deer's food. Only very small amounts of grasses were eaten ( $4 \%$ of volume), and of these Festuca rubra L. and Poa L. sp. were most often found.

\section{Summer Period (June 22nd - Sept. 21st)}

The material examined was obtained from roe-deer shot between July 21st and September 21st (Table 2). The rumens contained from 11 to 37 species of plants, a total of 97 being identified. During summer the total percentage of trees in the animals' food decreased. The most important species continued to be Quercus robur L., but its predominance over the other species of trees decreased. The leaves of Salix caprea L. and leaves and seed catkins of Betula verrucosa Ehrh. occurred extremely constantly. The chief role in the food of roe-deer in summer is played by shrubs and bush-like plants, Rubus idaeus L. being the most important. The stalks, leaves and berries of Vaccinium myrtilus L. also occurred in considerable amounts and sporadically also Vaccinium uliginosum L. In the rumens obtained in September decidedly predominated Calluna vulgaris (L.) Salisb. The amount of herb species in the total amount of food decreased, but the percentage of grasses and sedge increased, although they still formed only a small percentage of the food. A new component of the roe-deer's food appeared in the form of fungus, mainly of the Agaricaceae family.

\section{Autumn Period (Sept. 22nd-December 21st)}

Samples were examined from roe-deer shot between October 8th and December 17th (Table 2). A total of 63 species of plants were identified. The various rumens contained from 15 to 30 species of plants. During this period food from trees still forms a considerable part i.e. $20 \%$ of the rumen contents. Large amounts of yellowed leaves of Betula verrucosa Ehrh., Alnus glutinosa (L.) Gaertn., Populus tremula L., Salix caprea L., Salix pentandra L., Salix alba L., Tilia cordata Mill., Carpinus betulus L. were found in the rumens. Quercus sp. occurred chiefly in the form of twigs, acorns and galls, leaves being found only sporadically.

Shrubs and dwarf-shrubs formed the basis food. Among these the most numerously represented were Calluna vulgaris (L.) Salisb., Rubus idaeus L. and Vaccinium myrtillus L. In addition to Calluna vulgaris (L.) Salisb. the occurrence in the roe-deer's food of the leaves of Alnus glutinosa (L.) Gaertn., and plants typical of the habitats of this species, were characteristic of the autumn period. This was due to the śpecific 
Table 2.

Particulars of rumen contents of roe-deer at different seasons of the year.

\begin{tabular}{|c|c|c|c|}
\hline species & $\begin{array}{c}\text { Average } \\
\text { volume, } \\
\%\end{array}$ & $\begin{array}{c}\text { Freque- } \\
\text { ncy; } \%\end{array}$ & $\begin{array}{c}\text { Index of } \\
\text { importa- } \\
\text { nce } \%\end{array}$ \\
\hline \multicolumn{4}{|c|}{ SPRING } \\
\hline \multicolumn{4}{|c|}{ TREES } \\
\hline 1. Quercus L. sp.* & 27.1 & 100.0 & 2711 \\
\hline 2. Carpinus betulus L. & 2.8 & 77.8 & 216 \\
\hline 3. Populus tremula L. & 1.9 & 77.8 & 146 \\
\hline 4. Salix caprea L. & 2.1 & 66.7 & 141 \\
\hline 5. Betula verrucosa Ehrh. & 2.1 & 44.4 & 94 \\
\hline 6. Sorbus aucuparia L. & 1.3 & 44.4 & 59 \\
\hline 7. Acer platanoides L. & 0.4 & 33.3 & 15 \\
\hline 8. Tilia cordata Mill. & 0.1 & 44.4 & 5 \\
\hline 9. Malus silvestris (L.) Mill. & 0.1 & 11.1 & 1 \\
\hline 10. Pinus silvestris L. & + & 22.2 & + \\
\hline 11. Betula pubescens Ehrh. & + & 11.1 & + \\
\hline 12. Picea excelsa (Lam.) L. K. & + & 11.1 & + \\
\hline 13. Pirus communis L. & + & 11.1 & + \\
\hline 14. Salix aurita L. & + & 11.1 & + \\
\hline 15. Salix L. sp. & + & 11.1 & + \\
\hline
\end{tabular}

SHRUBS AND DWARF-SHRUBS

1. Rubus idaeus L.

2. Rubus saxatilis L.

3. Vaccinium myrtillus $\mathrm{L}$.

4. Corylus avellana L.

5. Crataegus L. sp.

6. Vaccinium vitis-idaea L.

7. Rosa L. sp.

8. Calluna vulgaris (L.) Salisb.

9. Prunus spinosa L.

10. Caragana arborescens Lam.

11. Sambucus nigra L.

\begin{tabular}{rrr}
2.9 & 77.8 & 225 \\
0.9 & 77.8 & 69 \\
0.5 & 66.7 & 37 \\
0.2 & 66.7 & 15 \\
0.3 & 33.3 & 11 \\
0.4 & 11.1 & 5 \\
0.1 & 33.3 & 4 \\
+ & 33.3 & + \\
+ & 11.1 & + \\
+ & 11.1 & + \\
+ & 11.1 & + \\
\hline
\end{tabular}

HERBS

$\begin{array}{lllr}\text { 1. Fragaria vesca L. } & 4.1 & 66.7 & 274 \\ \text { 2. Melampyrum pratense L. } & 3.6 & 66.7 & 237 \\ \text { 3. Chamaenerion angustifolium (L.) Scop. } & 5.0 & 44.4 & 222 \\ \text { 4. Potentilla erecta (L.) Hampe } & 2.8 & 77.8 & 216 \\ \text { 5. Rumex acetosella L. } & 2.9 & 44.4 & 128 \\ \text { 6. Ranunculus repens L. } & 3.4 & 22.2 & 76 \\ \text { 7. Hieracium L. sp. } & 1.1 & 66.7 & 74 \\ \text { 8. Ajuga reptans L. } & 4.4 & 11.1 & 49 \\ \text { 9. Veronica chamaedrys L. } & 0.5 & 55.7 & 31 \\ \text { 10. Geranium sanguineum L. } & 1.3 & 22.2 & 30 \\ \text { 11. Rumex acetosa L. } & 1.1 & 22.2 & 25 \\ \text { 12. Solidago virga-aurea L. } & 0.4 & 55.6 & 24 \\ \text { 13. Anthriscus silvestris (L.) Hoff. } & 0.9 & 22.2 & 20 \\ \text { 14. Pimpinella saxifraga L. } & 0.4 & 44.4 & 20 \\ \text { 15. Plantago lanceolata L. } & 0.7 & 22.2 & 15\end{array}$

*) Species of oak was not determined, but $Q$. robur occur most freguently in study area. Q. sessilis is rather rear and $Q$. rubra is planted sporadically. 
16. Geum rivale L.

17. Galium verum $\mathrm{L}$.

18. Potentilla anserina L.

19. Medicago lupulina L.

20. Trifolium repens $\mathrm{L}$.

21. Geum urbanum L.

22. Sonchus arvensis L.

23. Lathyrus pratensis L.

24. Galium mollugo L.

25. Alchemilla micans Bus.

26. Stellaria media Vill.

27. Astragalus glycyphyllos L.

28. Angelica silvestris L.

29. Geranium pratense L.

30. Ranunculus bulbosus L.

31. Galium L. sp.

32. Vicia L. sp.

33. Campanula patula L.

34. Melilotus albus Med.

35. Potentilla reptans $\mathrm{L}$.

36. Humulus lupulus L.

37. Galium vernum Scap.

38. Viola silvestris Rohb.

39. Veronica officinalis L.

40. Plantago maior L.

41. Alchemilla L. sp.

42. Senecio vernalis $\mathrm{W} . \mathrm{K}$.

43. Pulmonaria angustifolia L.

44. Hepatica nobilis Garsault

45. Centaurea jacea L.

46. Thymus serpyllum L.

47. Vicia dumetorum L

48. Knautia arvensis (L.) Coult

49. Polygonum aviculare L

50. Filipendula hexapetala Gilib.

51. Senecio L. sp.

52. Agrimonia eupatoria L.

53. Lamium purpureum L.

54. Trifolium alpestre $\mathrm{L}$.

55. Convallaria maialis $\mathrm{L}$.

56. Lotus corniculatus L.

57. Geranium L. sp.

58. Mycelis muralis (L.) Dum.

59. Trifolium L sp.

60. Scorzonera humilis L.

61. Daucus carota L.

62. Majanthemum bifolium (L.) F.W.Schm.

63. Isopyrum thalictroides L.

64. Thalictrum aquilegifolium $\mathrm{L}$.

$\begin{array}{llr}0.7 & 22.2 & 15 \\ 0.5 & 22.2 & 12 \\ 0.4 & 22.2 & 10 \\ 0.2 & 44.4 & 10 \\ 0.3 & 22.2 & 7 \\ 0.5 & 11.1 & 6 \\ 0.5 & 11.1 & 6 \\ 0.5 & 11.1 & 6 \\ 0.2 & 22.2 & 5 \\ 0.4 & 11.1 & 5 \\ 0.2 & 22.2 & 5 \\ 0.1 & 33.3 & 4 \\ 0.3 & 11.1 & 4 \\ 0.3 & 11.1 & 4 \\ 0.2 & 11.1 & 2 \\ 0.1 & 22.2 & 2 \\ 0.1 & 22.2 & 2 \\ 0.1 & 22.2 & 2 \\ 0.1 & 22.2 & 2 \\ 0.1 & 11.1 & 1 \\ 0.1 & 11.1 & 1 \\ 0.1 & 11.1 & 1 \\ 0.1 & 11.1 & 1 \\ + & 11.1 & + \\ + & 22.2 & + \\ + & 22.2 & + \\ + & 22.2 & + \\ + & 22.2 & + \\ + & 11.1 & + \\ + & 11.1 & + \\ + & 11.1 & + \\ + & 11.1 & + \\ + & 11.1 & + \\ + & 11.1 & + \\ + & 11.1 & + \\ + & 11.1 & + \\ + & 11.1 & + \\ + & 11.1 & + \\ + & 11.1 & + \\ + & 11.1 & + \\ + & 11.1 & + \\ + & 11.1 & + \\ + & 11.1 & + \\ + & 11.1 & + \\ + & 11.1 & + \\ + & 11.1 & + \\ + & 11.1 & + \\ + & 11.1 & + \\ + & 11.1 & + \\ + & & \\ + & & + \\ + & & + \\ + & & + \\ + & & +\end{array}$

GRASSES, SEDGE, RUSH
1. Gramineae L. sp.
$1.3 \quad 22.2$
2. Festuca rubra L.
0.7
22.2
33.3
3. Poa L. sp.
4. Poa pratensis L.
5. Poa nemoralis L.
$0.3 \quad 33.3$
6. Festuca ovina L.
7. Avenastrum pubescens (Huds.) Opiz. 0.1
8. Festuca L. sp.
9. Carex L. sp.
10. Agropyron repens (L.) P. B.
11. Luzula D. C. sp. 
FUNGI

1. Agaricaceae sp.

$+\quad 11.1+$

MOSSES

1. Mnium sp.

$+\quad 11.1+$

OTHER

1. Ants

2. Beetles

$\begin{array}{lll}+ & 11.1 & + \\ + & 11.1 & +\end{array}$

SUMMER

TREES

1. Quercus L. sp.

2. Salix caprea L.

3. Betula verrucosa Ehrh.

4. Populus tremula L.

5. Alnus glutinosa (L.) Gaertn.

6. Betula pubescens Ehrh.

7. Carpinus betulus L.

8. Pinus silvestris

9. Malus domestica Borb.

10. Sorbus aucuparia L.

11. Acer platanoides L.

$\begin{array}{llll}4.0 & 66.7 & 267\end{array}$

$\begin{array}{lrr}4.0 & 66.7 & 267 \\ 3.2 & 77.8 & 250 \\ 4.2 & 55.6 & 234 \\ 1.0 & 55.6 & 56 \\ 1.4 & 33.3 & 48 \\ 1.3 & 33.3 & 44 \\ 0.3 & 55.6 & 18 \\ 0.4 & 33.3 & 15 \\ 0.4 & 11.1 & 5 \\ 0.2 & 11.1 & 2 \\ 0.1 & 11.1 & 1\end{array}$

SHRUBS AND DWARF-SHRUBS

1. Rubus idaeus L.

2. Calluna vulgaris (L.) Salisb.

$14.3 \quad 77.8$

1115

3. Vaccinium myrtillus I.

4. Vaccinium uliginosum $\mathrm{L}$.

5. Rubus L. sp.

6. Rubus saxatilis L.

7. Vaccinium vitis-idaea L.

8. Corylus avellana L.

9. Juniperus communis L.

10. Frangula alnus Mill.

11. Oxycoccus quadripetalus Gilib.

$\begin{array}{rrr}14.3 & 77.8 & 1115 \\ 11.4 & 44.4 & 508 \\ 6.0 & 77.8 & 467 \\ 10.0 & 22.2 & 222 \\ 4.9 & 33.3 & 163 \\ 1.0 & 55.6 & 56 \\ 1.9 & 22.2 & 42 \\ 0.5 & 22.2 & 12 \\ + & 11.1 & + \\ + & 11.1 & + \\ + & 11.1 & +\end{array}$

HERBS

1. Plantago lanceolata $\mathrm{L}$.

2. Galium L. sp.

3. Fragaria vesca L.

4. Rumex L. sp.

5. Geum urbanum L.

6. Melampyrum pratense L.

7. Potentilla erecta (L.) Hampe

8. Centaurea jacea L.

9. Plantago maior L.

10. Cirsium palustre (L.) Scop.

11. Solidago virga-aurea L.

12. Veronica chamaedrys $\mathrm{L}$.

13. Filipendula ulmaria (L.) Maxim.

14. Chamaenerion angustifolium (L.) Scop.

15. Taraxacum officinale $\mathrm{L}$.

16. Lathyrus vernus (L.) Bernh.

$\begin{array}{lrr}4.4 & 44.4 & 197 \\ 2.2 & 55.6 & 123 \\ 1.1 & 55.6 & 62 \\ 1.7 & 33.3 & 56 \\ 1.2 & 44.4 & 54 \\ 0.9 & 33.3 & 30 \\ 0.3 & 33.3 & 11 \\ 0.3 & 33.3 & 11 \\ 0.6 & 11.1 & 6 \\ 0.6 & 11.1 & 6 \\ 0.2 & 22.2 & 5 \\ 0.1 & 55.6 & 5 \\ 0.2 & 22.2 & 5 \\ 0.1 & 22.2 & 2 \\ 0.2 & 11.1 & 2 \\ 0.2 & 11.1 & 2\end{array}$


17. Ajuga reptans L.

18. Viola L. sp.

19. Hieracium L. sp.

20. Urtica dioica L.

21. Galeobdolon luteum Huds.

22. Anthriscus silvestris (L.) Hoff.

23. Potentilla reptans $\mathrm{L}$.

24. Geranium L. sp.

25. Alectorolophus glaber (Lam.) Beck.

26. Knautia arvensis (L.) Coult.

27. Vicia L. sp.

28. Oxalis acetosella $\mathrm{L}$.

29. Campanula patula L.

30. Epilobium palustre L.

31. Cirsium arvense (L.) Scop.

32. Veronica officinalis $\mathrm{L}$.

33. Stellaria graminea L.

34. Geranium sanguineum L.

35. Thymus serpyllum L.

36. Polygonum aviculare $\mathrm{L}$.

37. Polygala comosa Schkr.

38. Epilobium L. sp.

39. Geranium palustre L.

40. Rumex acetosa L.

41. Trifolium repens $\mathrm{L}$.

42. Stellaria media Vill.

43. Helianthemum ovatum (Viv. Dun.

44. Melilotus albus Med.

45. Agrimonia eupatoria L.

46. Ranunculus repens L.

47. Potentilla anserina $\mathrm{L}$.

48. Adoxa moschatellina L.

49. Aegopodium podagraria $\mathrm{L}$.

50. Humulus lupulus L.

51. Angelica silvestris L.

52. Lychnis flos-cuculi L.

GRASSES, SEDGE, RUSH

1. Festuca rubra L.

2. Poa pratensis L.

3. Carex L. sp.

4. Agrostis vulgaris With.

5. Poa annua L.

6. Poa compressa L.

7. Agropyron repens (L.) P. B.

11.1

8. Phleum pratense L.

9. Dactylis glomerata L.

10. Festuca pratensis Huds.

11. Bromus mollis L.

12. Calamagrostis arundinacea (L.) Roth.

13. Deschampsia caespitosa (L.) P. B.

14. Festuca ovina L.

15. Juncus conglomeratus L.

16. Poa L. sp.

17. Calamagrostis adans. sp.

18. Brachypodium silvaticum (Huds.) Roem. et Schult.

19. Deschampsia flexuosa (L.) Trin.

20. Graminea L. sp.

21. Koeleria glauca (Schkuhr.) DC.

22. Lolium multiflorum Lam.

23. Melica nutans L. 
FERNS, LYCOPODS

1. Cystopteris fragilis (L.) Bernh.

2. Equisetum silvaticum $\mathrm{L}$.

$\begin{array}{lll}+ & 11.1 & + \\ + & 22.2 & +\end{array}$

FUNGI

1. Agaricaceae sp.

1.9

33.3

63

MOSSES

1. Mnium sp.

0.1

22.2

2

AUTUMN

TREES

1. Pinus silvestris L

2. Betula verrucosa Ehrh.

3. Quercus L. sp.

4. Alnus glutinosa (L.) Gaertn.

5. Populus tremula L.

6. Carpinus betulus L.

7. Salix caprea L.

8. Tilia cordata Mill.

9. Salix pentandra L.

10. Acer platanoides L.

11. Picea excelsa (Lam.) L.K.

12. Sorbus aucuparia L.

13. Salix alba L.

14. Betula pubescens Ehrh.

\begin{tabular}{rrr}
4.9 & 85.7 & 539 \\
3.9 & 100.0 & 386 \\
1.6 & 85.7 & 135 \\
2.9 & 42.9 & 123 \\
1.7 & 57.1 & 98 \\
1.0 & 71.4 & 71 \\
1.6 & 42.9 & 67 \\
0.7 & 57.1 & 41 \\
0.7 & 14.3 & 10 \\
0.6 & 14.3 & 8 \\
0.1 & 42.9 & 6 \\
0.1 & 28.6 & 4 \\
0.1 & 14.3 & 2 \\
+ & 42.9 & + \\
\hline
\end{tabular}

SHRUBS AND DWARF-SHRUBS

1. Calluna vulgaris (L.) Salisb.

2. Rubus idaeus I.

3. Vaccinium myrtillus L.

4. Vaccinium vitis-idaea L.

5. Corylus avellana L.

6. Rubus saxatilis L.

7. Rubus L. sp.

8. Juniperus communis L.

9. Rhammus cathartica L.

10. Frangula alnus Mill.

11. Rosa rubiginosa L.

$\begin{array}{rrr}22.0 & 71.4 & 1571 \\ 8.4 & 85.7 & 723 \\ 6.9 & 85.7 & 588 \\ 5.4 & 42.9 & 233 \\ 0.1 & 42.9 & 6 \\ 0.1 & 28.6 & 4 \\ 0.1 & 14.3 & 2 \\ + & 14.3 & + \\ + & 14.3 & + \\ + & 14.3 & + \\ + & 14.3 & +\end{array}$

HERBS

1. Hepatica nobilis Garsault

2. Brassica oleracea L. var. acephala DC.

$\begin{array}{rrr}3.6 & 42.9 & 153 \\ 6.4 & 14.3 & 92 \\ 2.0 & 42.9 & 86 \\ 2.4 & 28.6 & 69 \\ 1.1 & 57.1 & 65 \\ 2.1 & 28.6 & 61 \\ 2.1 & 28.6 & 61 \\ 0.4 & 57.1 & 25 \\ 0.4 & 42.9 & 18 \\ 0.4 & 42.9 & 18 \\ 0.6 & 28.6 & 16 \\ 0.3 & 42.9 & 12\end{array}$

3. Fragaria vesca L

4. Cirsium arvense (L.) Scop.

5. Solidago virga-aurea L.

6. Geum rivale L.

7. Urtica dioica L

8. Veronica chamaedrys L.

9. Oxalis acetosella L.

10. Mycelis muralis (L.) Dum.

11. Veronica officinalis $\mathrm{L}$

12. Lathyrus vernus (L.) Bernh.

$0.3 \quad 42.9$ 
13. Galium L. sp.

14. Sonchus arvensis L.

15. Hypericum perforatum L.

16. Stellaria media Vill.

17. Lamium album $\mathrm{L}$.

18. Succisa pratensis Mnch.

19. Hieracium L. sp.

20. Plantago maior L.

21. Rumex L. sp.

22. Trifolium repens $\mathrm{L}$.

23. Taraxacum officinalis $\mathrm{L}$.

24. Trifolium alpestre $\mathrm{L}$.

25. Galeopsis L. sp.

26. Ajuga reptans L.

27. Viola L. sp.

28. Glechoma hederasea L.

$\begin{array}{lll}0.3 & 28.6 & 8 \\ 0.4 & 14.3 & 6 \\ 0.3 & 14.3 & 4 \\ 0.1 & 14.3 & 2 \\ 0.1 & 14.3 & 2 \\ 0.1 & 14.3 & 2 \\ + & 14.3 & + \\ + & 14.3 & + \\ + & 14.3 & + \\ + & 28.6 & + \\ + & 28.6 & + \\ + & 14.3 & + \\ + & 14.3 & + \\ + & 14.3 & + \\ + & 14.3 & + \\ + & 14.3 & +\end{array}$

GRASSES, SEDGE, RUSH

1. Festuca rubra L.

2. Poa pratensis L.

3. Gramineae L. sp.

4. Calamagrostis arundinacea (L.) Roth.

5. Festuca ovina L.

6. Festuca L. sp.

7. Deschampsia caespitosa (L.) P.B.

8. Carex L. sp.

9. Agrostis vulgaris With.

10. Luzula L. sp.

\begin{tabular}{rrr}
0.3 & 42.9 & 12 \\
0.3 & 28.6 & 8 \\
0.6 & 14.3 & 8 \\
0.3 & 14.3 & 4 \\
0.1 & 28.6 & 4 \\
+ & 14.3 & + \\
+ & 14.3 & + \\
+ & 14.3 & + \\
+ & 14.3 & + \\
+ & 14.3 & + \\
\hline
\end{tabular}

FUNGI

1. Agaricaceae sp.

3.0

28.6

86

MOSSES

1. Hylocomium sp.

$+\quad 14.3+$

WINTER

TREES

1. Pinus silvestris L.

2. Picea excelsa (Lam.) L.K.

3. Betula verrucosa Ehrh.

4. Quercus L. sp.

5. Carpinus betulus $\mathrm{L}$.

6. Alnus glutinosa (L.) Gaertn.

7. Tilia cordata Mill.

8. Populus tremula L.

9. Larix decidua Mill.

10. Salix pentandra L.

11. Sorbus aucuparia L.

12. Salix caprea L.

13. Salix L. sp.

$\begin{array}{rrr}29.9 & 100.0 & 2990 \\ 6.9 & 57.1 & 394 \\ 1.3 & 80.9 & 104 \\ 1.4 & 47.6 & 66 \\ 1.1 & 57.1 & 65 \\ 1.1 & 47.6 & 54 \\ 0.2 & 33.3 & 8 \\ 0.2 & 23.8 & 6 \\ 0.2 & 23.8 & 5 \\ 0.3 & 9.5 & 3 \\ 0.1 & 28.6 & 3 \\ 0.1 & 14.3 & 1 \\ + & 9.5 & +\end{array}$

SHRUBS AND DWARF-SHRUBS

1. Calluna vulgaris (L.) Salisb.

2. Vaccinium myrtillus L.

17.5

85.7

1498 
3. Vaccinium vitis-idaea $\mathrm{L}$.

4. Juniperus communis L.

5. Rubus idaeus L.

6. Rubus L. sp.

7. Corylus avellana L

8. Crataegus L. sp.

9. Rhamnus catartica L.

10. Rosa L. sp.

11. Andromeda polifolia L.

$71.4 \quad 218$

$66.7 \quad 67$

$33.3 \quad 10$

$19.1 \quad 6$

$33.3 \quad 3$

19.1

4.8

4.8

4.8

HERBS

1. Fragaria vesca $\mathrm{L}$.

$23.8 \quad 71$

2. Medicago sativa L.

3. Oxalis acetosella L.

4. Veronica officinalis L.

5. Cerastium L. sp.

6. Solidago virga-aurea L.

7. Cirsium arvense (L.) Scop.

8. Carlina vulgaris L.

9. Veronica chamaedrys L.

10. Trifolium repens $\mathrm{L}$.

11. Stellaria media Mill.

12. Urtica dioica L.

13. Galium L. sp.

14. Erophila verna (L.) C.A.M.

15. Silene nutans L.

16. Galium Schultesi Vest.

17. Potentilla reptans $\mathrm{L}$.

18. Lamium album L.

19. Lotus corniculatus L.

20. Hypochoeris radicata $\mathrm{L}$.

21. Ajuga reptans L.

22. Cirsium oleraceum (L.) Scop.

23. Stellaria L. sp.

24. Hepatica nobilis Garsault

25. Geum rivale L.

26. Hieracium L. sp.

27. Geum urbanum L.

28. Bellis perennis L.

29. Papilionaceae

30. Hieracium Lachenalii Gmel.

31. Hypericum perforatum L.

32. Potentilla erecta (L.) Hampe

33. Melampyrum pratense L.

34. Lathyrus vernus (L.) Bernh.

35. Stellaria holostea L.

36. Labiatae sp.

37. Geranium L. sp.

38. Helianthemum ovatum (Viv.) Dun.

39. Trifolium pratense $\mathrm{L}$.

40. Centaurea cyanus L.

41. Polygonum convolvulus L.

42. Polygonum dumetorum $\mathrm{L}$.

43. Hieracium flagellare Willd.

$\begin{array}{rrr}3.0 & 23.8 & 71 \\ 3.1 & 9.5 & 30 \\ 0.7 & 33.3 & 22 \\ 0.6 & 19.1 & 16 \\ 0.4 & 14.3 & 7 \\ 0.2 & 23.8 & 5 \\ 0.2 & 23.8 & 5 \\ 0.4 & 9.5 & 4 \\ 0.2 & 9.5 & 2 \\ 0.2 & 4.8 & 1 \\ 0.1 & 19.1 & 1 \\ 0.1 & 14.3 & 1 \\ 0.1 & 14.3 & 1 \\ 0.1 & 4.8 & 1 \\ 0.1 & 9.5 & + \\ 0.1 & 4.8 & + \\ 0.1 & 4.8 & + \\ 0.1 & 4.8 & + \\ 0.1 & 4.8 & + \\ 0.1 & 4.8 & + \\ 0.1 & 4.8 & + \\ 0.1 & 4.8 & + \\ + & 4.8 & + \\ + & 23.8 & + \\ + & 14.3 & + \\ + & 14.3 & + \\ + & 9.5 & + \\ + & 9.5 & + \\ + & 9.5 & + \\ + & 4.8 & + \\ + & 4.8 & + \\ + & 4.8 & + \\ + & 4.8 & + \\ + & 4.8 & + \\ + & 4.8 & + \\ + & 4.8 & + \\ + & 4.8 & + \\ + & 4.8 & + \\ + & 4.8 & + \\ + & 4.8 & + \\ + & 4.8 & + \\ + & 4.8 & + \\ + & 4.8 & + \\ + & & +\end{array}$

GRASSES, SEDGE, RUSH
1. Dactylis glomerata L.
1. Deschampsia caespitosa (L.) P.B.
0.6
23.8
3. Poa pratensis L. 


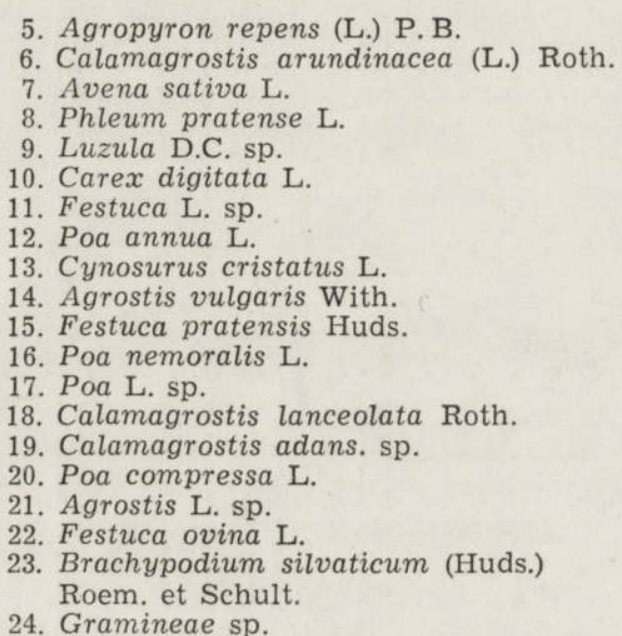

$\begin{array}{rrr}0.1 & 9.5 & 1 \\ 0.1 & 28.6 & 1 \\ 0.3 & 4.8 & 1 \\ 0.2 & 4.8 & 1 \\ 0.1 & 23.8 & 1 \\ 0.1 & 9.5 & + \\ 0.1 & 9.5 & + \\ 0.1 & 4.8 & + \\ 0.1 & 9.5 & + \\ 0.1 & 9.5 & + \\ 0.1 & 4.8 & + \\ 0.1 & 4.8 & + \\ + & 14.3 & + \\ + & 9.5 & + \\ + & 9.5 & + \\ + & 4.8 & + \\ + & 4.8 & + \\ + & 4.8 & + \\ + & 4.8 & + \\ + & 4.8 & +\end{array}$

FERNS, LYCOPODS

1. Dryopteris spinulosa (Mill.) O. Kuntze 2. Pteridium aquilinum (L.) Kuhn

$\begin{array}{lrl}0.3 & 42.9 & 14 \\ 0.1 & 4.8 & +\end{array}$

FUNGI

1. Polyporaceae sp. 2. Agaricaceae sp.

$\begin{array}{lll}+ & 14.3 & + \\ + & 14.3 & +\end{array}$

MOSSES

1. Mnium sp.

$+\quad 14.3+$

OTHER

1. Oakgall

$+9.5+$

microclimmate of the habitats of Alnus glutinosa (L.) Gaertn. which prolongs the growing period of plants.

Fungi formed about $3 \%$ of the total volume of the food. In addition food planted for deer, mainly Brassica oleracea var. acephala DC. were found.

\section{Winter Period (December 22nd - March 21st)}

Examination was made of the rumen contents of roe-deer shot between December 22nd and February 10th (Table 2).

The content of rumen was more constant in respect of species composition. Pinus silvestris L. occurred in all the rumens obtained 
during the winter, and occupied on an average $30 \%$ of the volume of the rumen, and in sporadic cases exceeded $60 \%$. The state of the particles examined showed that the roe-deer bites or browse the pine needles. It is, however, rarely that they bite off the needles together with the terminal parts of branches, and in such cases more often bite off the lateral shoots than the ones at the top. This does not mean of course, that roe-deer do not do any damage in young tree plantations. Picea excelsa (Lam) Lk. occurred in half of the rumens and occupied about $7 \%$ of their volume. In 5 rumens $1 \%$ of volume was occupied by branches of Larix decidua Mill. The browse of deciduous trees occurred in small amounts only, the following being most often found: Betula verrucosa Ehrh., Quercus L. sp., Carpinus betulus L., Alnus glutinosa (L.) Gaertn; fallen leaves were found in the rumens in addition to twigs. Trees supplied about $43 \%$ of the animals' winter food.

Shrubs and dwarf-shrubs played an important part in the winter food of roe-deer. Calluna vulgaris (L.) Salisb. occurred in $86 \%$ of the rumens examined, in amounts of about $17 \%$ of the rumen contents. In addition to leaves, bark and xylem, this plant supplied a considerable amount of dried seeds in the bracts of the perianths. Vaccinium myrtillus L. occurred less often, but nevertheless in considerable amounts, and in $71 \%$ of the rumens in amounts of about $3 \%$ Vaccinium vitis-idaea L. Many rumens contained the needles, ends of shoots and berries of Juniperus communis $\mathrm{L}$. in amounts of about $1 \%$ of the rumen contents.

The roe-deer ate small amounts of green herbs and their seeds throughout the winter 43 of such species were indentified, forming about $10 \%$ of the animals' food. One of the most important was Fragaria vesca L., occuring in quantities of approximately $3 \%$ in one quarter of the rumens examined. Grasses continued to form only a small percentage 2.3 - of the food. In addition ferns and fragments of fungi of the Polyporaceae and Agaricaceae families were found sporadically.

A total of 91 species of plants were found in rumen contents in winter. The various rumens contained from 9 to 36 of the species identified.

From one to three species of plants dominated in the rumen contents in each of the seasons investigated. This indicates that roe-deer have distinct preferences for given species at different seasons of the year. These observations agree with the data given by $\mathrm{Kom}$ a rek (1954) and Mottl (1957). In all the periods, however, the roe-deer's food was varied and the contents of individual rumens contained from 9 to 42 species of plants in different forms of growth habit. The results obtained justify the statement that the food supply of roe-deer living 
in a forest habitat are trees, shrubs, dwarf-shrubs and herbs. Grasses and sedges form only a very small percentage of their food (Table 3 ).

Trees form $38 \%$ of the food in spring, Quercus L. sp. in the form of leaves and shoots being the preferred species, while the other species of trees were consumed mainly in the form of leaves. The participation of trees in the roe-deer's food decreased in summer and autumn, and rose again in winter to $43 \%$. Pinus silvestris L., appeared in rumen contents in autumn, in winter it increased to an average $30 \%$ of the food.

Table 3.

Percentage of plant groups in the contents of the rumens examined at different seasons of the year.

\begin{tabular}{|c|c|c|c|c|}
\hline Seasons & $\begin{array}{c}\text { Spring } \\
\text { March 22- } \\
\text { June 21 }\end{array}$ & $\begin{array}{c}\text { Summer } \\
\text { June } 22- \\
\text { Sept. } 21\end{array}$ & $\begin{array}{l}\text { Autumn } \\
\text { Sept. 22- } \\
\text { Dec. } 21\end{array}$ & $\begin{array}{l}\text { Winter } \\
\text { Dec. 22- } \\
\text { March 21 }\end{array}$ \\
\hline No. of rumens examined & 9 & 9 & 7 & 21 \\
\hline Trees & 38 & 17 & 20 & 43 \\
\hline Shrubs and bushy plants & 5 & 50 & 43 & 37 \\
\hline Herbs & 39 & 15 & 23 & 11 \\
\hline Grasses, sedge, seeds & 4 & 7 & 2 & 2 \\
\hline Ferns, lycopods & - & + & - & + \\
\hline Fungi & + & 2 & 3 & + \\
\hline Mosses & + & + & + & + \\
\hline $\begin{array}{l}\text { Particles with } "+\ll \\
\text { and unidentified }\end{array}$ & 13 & 9 & 9 & 6 \\
\hline
\end{tabular}

Shrubs and dwarf-shrubs develop later than grasses and herbs, and therefore form only $5 \%$ of the food in spring, but in summer form as much as half of the rumen contents. Species of the genus Rubus and Vaccinium usually dominated. Calluna vulgaris (L.) Salisb. became one of the most important component in autumn and winter.

Herbs were eaten in considerable quantities in spring only, after which their participation in the food decreased. The following genera were preferred: Fragaria, Geum, Potentilla, Solidago, Hieracium, Cirsium, Melampyrum, Veronica, Ranunculus, Hepatica, Chamaenerion. The following genera of grasses and sedges occurred: Festuca, Poa, Dactylis, Deschampsia, Calamagrostis and Carex digitata L.

$\mathrm{K} l$ öt z li (1965) drew attention to the fact that roe-deer eat poisonous plants. His observations were confirmed by our findings. The following species, considered as poisonous, were found in the rumens we examined: Convallaria maialis L., Lilium martagon L., Ranunculus acer L., 
Ranunculus repens L., Hepatica nobilis (L.) Garsault., Melampyrum pratense $\mathrm{L}$.

The very small extent to which roe-deer feed on the abundant fodder laid out in feeding places for game is remarkable. It was only in two out of the 21 rumens examined in winter that fodder originating from supplementary feeding was found, i.e. Medicago sativa L., Dactylis glomerata L. and Lotus corniculatus L. It would thus appear that the basic fodder for supplementary feeding of roe-deer in winter should be good leaf fodder.

\section{CONCLUSIONS}

1. The food of roe-deer living in the Pisz Forest is very varied. The basis of their food is formed by leaves and browse of trees and shrubs and also dwarf-shrubs. Herbs play a considerable part in their food, especially in spring. Grasses are only of secondary importance from the aspect of quantity. Such food preferences result in roe-deer doing considerable damage to young tree plantations, particularly when the latter contain a certain proportion of deciduous trees, and to pine plantations in winter.

2. Roe-deer, on account of their feeding habits, must be given equal consideration with red deer in cases in which it is necessary to reduce the deer population.

3. Roe-deer make relatively little use of fodder laid out in the winter, whether meadow hay or papilionaceous fodder, and therefore it is necessary to provide them with food consisting mainly of good leaf fodder.

\section{REFERENCES}

1. Anderson A. E., Snyder W. A. \& Brown G. W., 1965: Stomach content analyses related to condition in mule deer, Guadalupe Mountains, New Mexico. J. Wildl. Mgmt., 29, 2: 352-366.

2. Chamrad A. D. \& Box T. W., 1964: A point frame for sampling rumen contents. J. Wildl. Mgmt., 28, 3: 473-477.

3. C zarnocki J., 1950: Klucz do oznaczania traw. Państw. Wyd. Roln. i Leśne: $1-347+59$. Warszawa.

4. G uziński L., 1964: Ciężar zwierzyny grubej na Mazurach. Łowiec Pol., 17 (1236): 14-15. Warszawa.

5. Klötzli F., 1965: Qualität und Quantität der Rehäsung in Wald und Grünland-Gesellschaften des nördlichen Schweizer Mittellandes. Diss. Dokt. Naturwiss. Eidgenöss. techn. Hochschule Zürich. 1-187. Zürich.

6. Kolev I., 1966: Proučvanija vrhu hraneneto na srnata v Cepinskija rajon. Gorskostopanska Nauka., 3, 1: 71-81. 
7. Ko marek J., 1954: Leśnicka zoologia. 3rd edition. Praha.

8. Kulesza W., 1952: Klucz do oznaczania drzew i krzewów. Państw. Wyd. Roln. i Leśne: 1-295. Warszawa.

9. M 0 t t 1 S., 1957: Potrava srnči zvèře (C.c.c.L.). Biologia, 12, 1: 29-42. Bratislava.

10. N e ča s J., 1963: Srnči zvèř: 77-87. Praha.

11. R a esfeld F. von, 1965: Das Rehwild, 6th edition: 12-13. Hamburg-Berlin.

12. Szafer W., Kulczyński S. \& Paw lowski B., 1953: Rośliny polskie. Państw. Wyd. Nauk.: XXVIII + 1-1020. Warszawa.

Received, January 22, 1969.

Polish Academy of Science,

Institute of Experimental Animal Breeding,

Popielno, pow. Pisz.

Andrzej SIUDA, Wirgiliusz ŻUROWSKI, Hanna SIUDA.

\section{POKARM SARNY}

\section{Streszczenie}

W ciągu czterech sezonów łowieckich w latach 1963-1966 zbadano 46 żwaczy saren (Capreolus capreolus L.). Zebrany materiał pochodził z części Puszczy Piskiej $\left(53^{\circ} 43^{\prime} \mathrm{N}, 21^{\circ} 36^{\prime} \mathrm{E}\right)$, gdzie dominowały zespoly Pineto-Vaccinietum myrtilli i Pineto-Quercetum. Zidentyfikowano 178 gatunków roślin. Stwierdzono sezonową zmienność pokarmu sarny (Tabele 2, 3). Podstawę pożywienia stanowily liście i pędy drzew, krzewów i krzewinek. W okresie wiosennym znaczną rolę odgrywały zioła. Trawy i turzyce występowały w niewielkich ilościach. W poszczególnych żwaczach rozpoznano od 9 do 42 gatunków roślin, z których jeden lub najwyżej trzy dominowały.

Wiosną dominowaly: Quercus sp., Fragaria vesca L., Melampyrum pratense L., Chamaenerion angustifolium (L.) Scop., Carpinus betulus L.; latem: Rubus idaeus L., Vaccinium myrtillus L., Quercus sp., Salix caprea L., Betula verrucosa Ehrh.; jesienią: Calluna vulgaris (L.) Salisb., Rubus idaeus L., Vaccinium myrtillus L., Pinus silvestris L., Betula verrucosa $\mathrm{Eh} \mathrm{hh.:} \mathrm{zimą} \mathrm{zaś:} \mathrm{Pinus} \mathrm{sil-}$ vestris L., Calluna vulgaris (L.) S a lis b.; Vaccinium myrtillus L., Picea excelsa (L a m.) Lk., Vaccinium vitis-idaea L. 